\title{
A bound on permutation codes
}

\author{
Jürgen Bierbrauer \\ Department of Mathematical Sciences \\ Michigan Technological University \\ Houghton, Michigan 49931, USA \\ jbierbra@mtu.edu
}

\author{
Klaus Metsch \\ Universität Gießen \\ Mathematisches Institut \\ Arndtstr. 2, D-35392 Gießen, Germany \\ klaus.metsch@math.uni-giessen.de
}

Submitted: Nov 28, 2012; Accepted: July 18, 2013; Published: July 26, 2013

Mathematics Subject Classifications: 05A05, 05B40, 51E26, 94B60

\begin{abstract}
Consider the symmetric group $S_{n}$ with the Hamming metric. A permutation code on $n$ symbols is a subset $C \subseteq S_{n}$. If $C$ has minimum distance $\geqslant n-1$, then $|C| \leqslant n^{2}-n$. Equality can be reached if and only if a projective plane of order $n$ exists. Call $C$ embeddable if it is contained in a permutation code of minimum distance $n-1$ and cardinality $n^{2}-n$. Let $\delta=\delta(C)=n^{2}-n-|C|$ be the deficiency of the permutation code $C \subseteq S_{n}$ of minimum distance $\geqslant n-1$.

We prove that $C$ is embeddable if either $\delta \leqslant 2$ or if $\left(\delta^{2}-1\right)(\delta+1)^{2}<27(n+2) / 16$. The main part of the proof is an adaptation of the method used to obtain the famous Bruck completion theorem for mutually orthogonal latin squares.
\end{abstract}

\section{Introduction}

Definition 1. Consider the symmetric group $S_{n}$ equipped with the Hamming distance. A permutation code is a subset $C \subseteq S_{n}$ and the minimum distance $d=d(C)$ is the minimum distance between two members of $C$.

Permutation codes are also known as permutation arrays and as permutation sets. There is a vast literature on the subject. Motivation comes from data transmission over power lines, see $[7,15,6,5]$, and the design of block ciphers [14]. A related and in some sense dual notion are uniformly $t$-homogeneous sets of permutations where the defining property is that there is a constant $\mu>0$ such that for any two not necessarily different unordered $t$-subsets $A$ and $B$ of letters the number of permutations $\pi \in C$ mapping $\pi: A \mapsto B$ equals $\mu$. Those sets have been studied in the framework of perpendicular arrays, see for example $[1,2,11]$.

Clearly a permutation code $C \subseteq S_{n}$ of distance $n$ has at most $n$ codewords, with equality if and only if $C$ is a Latin square of order $n$. The case of minimum distance $n-1$ leads to the following notion: 
Definition 2. A permutation code $C \subseteq S_{n}$ is sharply 2-transitive if for any two pairs $(a, b),(c, d)$ of symbols such that $a \neq b, c \neq d$ there is precisely one $\pi \in C$ such that $\pi(a)=b$ and $\pi(c)=d$.

Proposition 3. A permutation code $C \subseteq S_{n}$ of minimum distance $\geqslant n-1$ has size at most $n(n-1)$, with equality if and only if $C$ is sharply 2-transitive. A 2-transitive set of permutations on $n$ letters exists if and only if a projective plane of order $n$ exists. This is the case if $n$ is a prime-power.

Proof. Most of the claims are well-known and easy to see. For the sake of completeness we sketch the proof of equivalence between projective planes and sharply 2-transitive permutation sets. Given a projective plane of order $n$, choose a point $\infty$ and two lines $l_{1}, l_{2}$ on $\infty$. Label the points $\neq \infty$ on $l_{1}, l_{2}$ as $P_{1}, \ldots, P_{n}$ and $Q_{1}, \ldots, Q_{n}$, respectively. To each point $R \notin l_{1} \cup l_{2}$ associate a permutation $\pi_{R}$ such that $\pi_{R}(i)=j$ if $R, P_{i}, Q_{j}$ are collinear. The axioms of a projective plane show that this defines a sharply 2-transitive set of permutations. This proves one of the claims.

Let $C$ be a permutation code of distance $\geqslant n-1$. Associate to $C$ a geometry $\Pi(C)$ with $1+2 n+|C|$ points and $n^{2}+2$ lines where $l_{1}=\left\{\infty, P_{1}, \ldots, P_{n}\right\}$ and $l_{2}=\left\{\infty, Q_{1}, \ldots, Q_{n}\right\}$ are two of the lines, each $\pi \in C$ defines a point and for each pair $(i, j)$ where $1 \leqslant i, j \leqslant n$ the line $L_{i j}$ contains $P_{i}, Q_{j}$ and those $\pi \in C$ which map $\pi: i \mapsto j$. If $C$ is sharply 2transitive, then $\Pi(C)$ has $n^{2}+n+1$ points and $n^{2}+2$ lines and it is easy to see that it can be completed to a projective plane of order $n$.

In the sequel we will restrict attention to permutation codes $C \subset S_{n}$ of minimum distance $\geqslant n-1$.

Definition 4. A permutation code $C \subseteq S_{n}$ of minimum distance $\geqslant n-1$ is embeddable if it is contained in a sharply 2-transitive set. Let $M_{n}$ be the largest size of a permutation code $C \subseteq S_{n}$ of distance $\geqslant n-1$, and $m_{n}$ the largest size of a non-embeddable such code.

Observe that $M_{q}=q^{2}-q$ if $q$ is a prime-power, and $M_{n}=m_{n}<n^{2}-n$ provided $n$ is not the order of a projective plane. In those cases $m_{n}$ can be seen as a measure for the best possible approximation to the non-existing plane. The values of $m_{n}$ are known only for $n \leqslant 6$.

Result 5. $m_{4}=7, m_{5}=15, m_{6}=18$.

In fact in cases $n=2$ and $n=3$ the symmetric group is sharply 2-transitive itself, so $m_{n}$ is not defined in those cases. For $n=4$ it is easy to see that $m_{4}=7$. One such maximal non-embeddable code consists of the identity permutation and the elements of order 4. The value of $m_{5}$ is found in Bogaerts [3], the determination of $m_{6}$ is due to Kløve $[9,10]$.

Assume a set of $t$ mutually orthogonal Latin squares of order $n$ exists. The representation as a dual net shows immediately that this allows the construction of a permutation code $C \subseteq S_{n}$ of minimum distance $\geqslant n-1$ and size $t n$. This generalizes a statement from Proposition 3 which corresponds to case $t=n-1$. In particular the existence of 5 
mutually orthogonal Latin squares of order 12 shows $M_{12} \geqslant 60$. It is a recent result by $\mathrm{I}$. Janiszczak and R. Staszewski (personal communication) that $M_{12} \geqslant 112$.

Our main results are the following:

Theorem 6. Let $C \subseteq S_{n}$ be a permutation code of minimum distance $\geqslant n-1$, where $|C|=n^{2}-n-\delta$.

- If $\delta \leqslant 2$, then $C$ is embeddable.

- If $\left(\delta^{2}-1\right)(\delta+1)^{2}<27(n+2) / 16$, then $C$ is embeddable.

The embeddability of a permutation code of size $n^{2}-n-1$ (case $\delta=1$ of Theorem 6 ) had been shown by Quistorff [13]. Of particular interest is case $n=10$, the smallest integer $n>6$ for which no projective plane of order $n$ exists. The lower bound $M_{10} \geqslant 49$ has been shown in [8]. Theorem 6 shows $M_{10} \leqslant 87$.

Our proof is an adaptation of the celebrated Bruck embedding theorem for mutually orthogonal Latin squares, see $[4,12]$. In Section 2 we define a geometric representation of permutation sets. Some basic properties are proved in Section 3. The last two sections contain the proofs of the embeddability theorems.

\section{$2 \quad$ A geometric setting}

Definition 7. A permutation incidence structure of order $n$ consists of points and lines such that

- There are $n^{2}$ points.

- Each line has precisely $n$ points.

- Each point is on at least 2 lines.

- Two different lines meet in at most one point.

Definition 8. Let the total number of lines of a permutation incidence structure be $n^{2}+n-\delta$. Then $\delta$ is the deficiency and we write $P(n, \delta)$.

Observe that as a consequence of the last axiom, each point of a $P(n, \delta)$ is on at most $n+1$ lines. Permutation incidence structures are essentially geometric representations of permutation codes $C \subseteq S_{n}$. This geometry is obtained by dualizing the geometry $\Pi(C)$ used in the proof of Proposition 3. More precisely the following holds:

Proposition 9. The following are equivalent:

$A$ permutation code $C \subseteq S_{n}$ of minimum distance $\geqslant n-1$, where $|C|=n(n-1)-\delta$.

A $P(n, \delta)$ which possesses two parallel classes of lines each of which partitions the points. 
Proof. Let $C$ be a permutation code of distance $\geqslant n-1$ and size $n(n-1)-\delta$. The corresponding $P(n, \delta)$ is obtained from $\Pi(C)$ by omitting the point $\infty$ and dualization: the points of the $P(n, \delta)$ are the lines $L_{i j}$, the lines of the $P(n, \delta)$ are the permutations and the $2 n$ points $P_{i}, Q_{j}$ on the lines $l_{1}, l_{2}$.

Conversely let a $P(n, \delta)$ be given. The dual structure has $n^{2}$ lines. Each point is on $n$ lines, each line has at least 2 points and two different points are on at most one line. The additional property shows that this dual structure possesses two sets $\left\{P_{1}, \ldots, P_{n}\right\}$ and $\left\{Q_{1}, \ldots, Q_{n}\right\}$ of points with the property that each line contains exactly one of the $P_{i}$ and one of the $Q_{j}$. It follows that the lines are precisely the lines $L_{i j}$ through $P_{i}$ and $Q_{j}$. Let $X$ be one of the $n^{2}-n-\delta$ remaining points. Then $X$ determines a permutation on $n$ objects in the obvious way (the permutation maps $i \mapsto j$ if and only if $X \in L_{i j}$ ), The resulting permutation code $C$ has $n^{2}-n-\delta$ elements and minimum distance $\geqslant n-1$.

To sum up: each permutation code $C$ of minimum distance $\geqslant n-1$ and size $n^{2}-n-\delta$ defines a $P(n, \delta)$. On the other hand it is obvious that a $P(n, \delta)$ results if we remove some $\delta$ lines from an affine plane of order $n$. We want to prove that for small values of $\delta$ each $P(n, \delta)$ is embeddable in an affine plane. We are going to prove this if either $\delta \leqslant 2$ (Corollary 15 and Theorem 23) or $\left(\delta^{2}-1\right)(\delta+1)^{2}<27(n+2) / 16$ (Theorem 26). This will complete the proof of Theorem 6 .

\section{Basic properties of permutation incidence struc- tures}

In the sequel let $(\mathcal{P}, \mathcal{L})$ be a $P(n, \delta)$.

Definition 10. Let $r_{P}$ (the degree of $P$ ) be the number of lines on the point $P$ and $\delta_{P}=n+1-r_{P} \geqslant 0$ the deficiency of $P$. For a line $l$ define $\delta_{l}=\sum_{P \in l} \delta_{P}$, the deficiency of $l$. A point $P$ is exceptional if $\delta_{P}>0$. Let $E$ be the set of exceptional points. Lines $l, g$ are parallel if either $l=g$ or $l \cap g=\emptyset$. Let $i\left(l_{1}, l_{2}, \ldots\right)$ be the number of lines which are parallel to $l_{1}, l_{2}, \ldots$ and different from $l_{1}, l_{2}, \ldots$

Lemma 11. Let $P$ be a point and $l$ a line. The following hold:

- We have $r_{P} \leqslant n+1$ with equality if and only if $P$ is joined to all remaining points.

- If $P \notin l$ and $P$ non-exceptional, then $P$ is on precisely one line which is parallel to $l$.

- $\delta_{l} \geqslant 0$ with equality if and only if each $P \in l$ is non-exceptional.

- $i(l)=n-\delta-1+\delta_{l}$ for each line $l$.

Proof. The first statement is obvious (as $\left.1+(n+1)(n-1)=n^{2}\right)$. For the second statement observe that by the first $P$ is on precisely $n$ lines that are not parallel to $l$. It follows that precisely one line through $P$ must be parallel to $l$. The third claim follows from the first. 
As for the last claim, it is easier to count the lines which are not parallel to $l$. This number is $1+\sum_{P \in l}\left(r_{P}-1\right)=1+\sum_{P \in l}\left(n-\delta_{P}\right)=1+n^{2}-\delta_{l}$. Subtracting this from the total number $n^{2}+n-\delta$ of lines yields the result.

Lemma 12. We have $|E| \leqslant \sum_{P \in \mathcal{P}} \delta_{P}=\delta n$, with equality on the left if and only if all points have deficiency $\leqslant 1$.

Proof. Double counting of point-line incidences shows

$$
n\left(n^{2}+n-\delta\right)=n|\mathcal{L}|=\sum_{P \in \mathcal{P}} r_{P}=\sum_{P \in \mathcal{P}}\left(n+1-\delta_{P}\right)=n^{2}(n+1)-\sum_{P \in \mathcal{P}} \delta_{P}
$$

The equality follows by comparison, the inequality is an obvious consequence.

In particular $\delta \geqslant 0$ and $P(n, 0)$ is an affine plane. We can assume $\delta>0$ in the sequel. Here is a refinement of the preceding lemma:

Lemma 13. For each line $l$ the following hold:

- $|E \cap l| \leqslant \delta_{l}$, with equality if all points on l have deficiency $\leqslant 1$.

- $|E \backslash l| \leqslant \sum_{Q \in E \backslash l} \delta_{Q}=\delta n-\delta_{l}$ with equality if all points off $l$ have deficiency $\leqslant 1$.

Proof. Let $e_{l}=|E \cap l|$. The number of lines meeting $l$ in a point equals $\sum_{P \in l}\left(n-\delta_{P}\right)=$ $n^{2}-\delta_{l}$. On the other hand this number is $\leqslant\left(n-e_{l}\right) n+e_{l}(n-1)=n^{2}-e_{l}$. It follows $e_{l} \leqslant \delta_{l}$ with equality if every exceptional point of $l$ has deficiency one. The remaining statements are implied by Lemma 12.

Lemma 14. We have $\delta_{P} \leqslant \delta$ for all P. Equality holds if and only if all points collinear with $P$ are non-exceptional and all remaining points except $P$ have deficiency 1.

Proof. Let $P$ be an exceptional point. It is not collinear with $\delta_{P}(n-1)$ points and those points are exceptional. Let $l$ be a line on $P$. By Lemma 13 we have

$$
\delta_{P}(n-1) \leqslant|E \backslash l| \leqslant \delta n-\delta_{l} \leqslant \delta n-\delta_{P} .
$$

Comparison shows $\delta_{P} \leqslant \delta$. Equality holds if and only if all three inequalities above are met with equality.

Corollary 15. Each $P(n, 1)$ can be embedded in an affine plane.

Proof. There is an exceptional point $P$. By Lemma 14 there are precisely $1+n-1=n$ exceptional points and those are pairwise not collinear. Their union can be used as an additional line which completes the $P(n, 1)$ to an affine plane.

In the sequel we may assume $\delta \geqslant 2$.

Lemma 16. Let lines $l, h$ meet in an exceptional point. Then $i(l, h) \leqslant \delta_{l}+\delta_{h}-(1+\delta)$. 
Proof. We know $i(l)$. As $h$ contains at least $n-\delta_{h}$ non-exceptional points and each of those is on a line parallel to $l$, the number in question is bounded by the difference $i(l)-\left(n-\delta_{h}\right)=\delta_{l}+\delta_{h}-1-\delta$.

Lemma 17. Let lines $l \neq h$ be parallel, $\delta_{l}, \delta_{h}>0$. Then $i(l, h) \geqslant n-1-\delta-\left(\delta_{l}-1\right)\left(\delta_{h}-1\right)$.

Proof. Start from the $i(l)$ lines parallel to $l$ and different from $l$. How many of those intersect $h$ ? Each of the $\geqslant n-\delta_{h}$ non-exceptional points on $h$ contributes only $h$ itself, and each of the $\leqslant \delta_{h}$ exceptional points, being collinear with the non-exceptional points on $l$, contributes at most $\delta_{l}$ such lines one of which is $h$. It follows that the number in question is $\geqslant i(l)-1-\delta_{h}\left(\delta_{l}-1\right)$.

Lemma 18. If $h$ and $h^{\prime}$ meet, are both parallel to $l$ and $\delta_{l}, \delta_{h}, \delta_{h^{\prime}}>0$, then $n+1 \leqslant$ $\delta_{l}\left(\delta_{h}+\delta_{h^{\prime}}-1\right)$.

Proof. $h \cap h^{\prime}$ is an exceptional point as it is on two lines parallel to $l$. We have

$$
i(l) \geqslant 2+i(l, h)+i\left(l, h^{\prime}\right)-i\left(l, h, h^{\prime}\right) .
$$

We know $i(l)$, have lower bounds on $i(l, h), i\left(l, h^{\prime}\right)$ and an upper bound on $i\left(l, h, h^{\prime}\right) \leqslant$ $i\left(h, h^{\prime}\right)-1$. Comparing the extremes of these inequalities yields the claim.

Definition 19. For each line $l$, let $\Pi(l)$ the set of lines parallel to $l$.

We know from Lemma 11 that $\Pi(l)$ is a family of $n+\delta_{l}-\delta$ lines.

Lemma 20. Suppose $l$ is a line with at least $n-1$ non-exceptional points. Then $\Pi(l)$ consists of mutually parallel lines, and $\delta_{g} \geqslant \delta_{l}$ for all $g \in \Pi(l)$.

Proof. Let $l \neq g \in \Pi(l)$. We know that each non-exceptional point of $g$ is on precisely one line which is parallel to $l$. The presence of $n-1$ non-exceptional points on $l$ shows that the same is true for each exceptional point of $g$. It follows that the lines in $\Pi(l)$ are mutually parallel and therefore $\Pi(l) \subseteq \Pi(g)$. This implies $\delta_{l} \leqslant \delta_{g}$.

\section{Special cases}

Proposition 21. Each $P(n, \delta)$ containing a line of deficiency 0 and such that $n \geqslant \delta(2 \delta-$ 1) is embeddable.

Proof. Let $\delta_{l}=0$. Then $|\Pi(l)|=n-\delta$. Each point covered by a line from $\Pi(l)$ is on $n+1$ lines and therefore non-exceptional. It follows that those points are precisely the non-exceptional points. Each point outside is exceptional. Lemma 12 implies that the points not covered by $\Pi(l)$ form the set $E$ of exceptional points, each of deficiency $\delta_{Q}=1$. This implies that every line $l^{\prime} \in \Pi(l)$ satisfies $\delta_{l^{\prime}}=0$ and hence $\Pi\left(l^{\prime}\right)=\Pi(l)$. Let $g \notin \Pi(l)$. Then $g$ has precisely $n-\delta$ non-exceptional points, and therefore $\delta_{g}=\delta$. Hence, every line has deficiency 0 or $\delta$. 
Let $Q \in E$ and $U \subset E$ the $n$-set consisting of $Q$ and all points not collinear with $Q$. Assume some two points of $U$ are collinear on a line $h$. Then $Q$ is on some two different lines both of which are parallel to $h$. As all lines involved have deficiency $\delta$, then Lemma 18 yields a contradiction. This shows that $U$ can be added to the list of lines resulting in a $P(n, \delta-1)$ which still contains a line of deficiency 0 . We are done by induction.

Proposition 22. If $\delta>0$ and $n \geqslant(\delta-1)(2 \delta-3)$, then each $P(n, \delta)$ containing a point of deficiency $\delta$ is embeddable.

Proof. Let $\delta_{P_{0}}=\delta$. We are in the situation of Lemma 14 when equality holds. The bundle of lines $l_{1}, \ldots, l_{n+1-\delta}$ on $P_{0}$ covers $P_{0}$ and the $(n+1-\delta)(n-1)$ non-exceptional points. The complement is the set $N$ of $\delta(n-1)$ points of deficiency 1. We have $E=\left\{P_{0}\right\} \cup N$.

As $\left|\Pi\left(l_{i}\right)\right|=n$ it follows from Lemma 20 that the lines of $\Pi\left(l_{i}\right)$ partition the point set into $n$ lines. As each $g \in \Pi\left(l_{i}\right)$ satisfies $\delta_{g} \geqslant \delta$ (Lemma 20) and $\sum_{g \in \Pi\left(l_{i}\right)} \delta_{g}=\sum_{P \in \mathcal{P}} \delta_{P}=$ $\delta n$ it follows that $\delta_{g}=\delta$ and therefore $\Pi(g)=\Pi\left(l_{i}\right)$. Each of the remaining lines meets each of the $l_{i}$, each line parallel to $l_{i}$ and has deficiency $\delta-1$.

Let $X \in N$ and $U$ the set consisting of $X$ and the points not collinear with $X$. Clearly $P_{0} \in U$. As $\delta_{X}=1$ we have $|U|=n$. Assume a line $h$ contains two points from $U$. As $X$ has degree $n$, it is on at least two lines parallel to $h$. The first part of the proof shows that $h$ and the two lines on $X$ parallel to $h$ have deficiency $\delta-1$. Lemma 18 yields a contradiction. It follows that $U$ can be added as a line to produce a $P(n, \delta-1)$, in which $P_{0}$ is a point of deficiency $\delta-1$. We are done by induction.

Theorem 23. Each $P(n, 2)$ is embeddable.

Proof. For $n \leqslant 6$ this is known. Assume therefore $n>6$. Because of Proposition 21 and Proposition 22 it can be assumed that all lines have positive deficiency (equivalently: contain at least one exceptional point) and $\delta_{P} \leqslant 1$ for all $P$. The latter implies $|E|=$ $\sum_{P \in \mathcal{P}} \delta_{P}=2 n$. As the removal of two lines from an affine plane of order $n$ produces a $P(n, 2)$ which either has a line of deficiency 0 or has a point of deficiency 2 , we expect a contradiction.

Considering the distribution of the $2 n$ exceptional points on the bundle of lines on a non-exceptional point $P$, we see that $P$ is on a line $l$ of deficiency 1 . We have $|\Pi(l)|=n-1$. Lemma 20 shows that the lines of $\Pi(l)$ are parallel. They cover $n(n-1)$ points. As the $n$ exceptional points covered by $\Pi(l)$ distribute on the $n-1$ lines of this partial parallel class, there is precisely one line $g \in \Pi(l)$ of deficiency 2 . We have $\Pi\left(l^{\prime}\right)=\Pi(l)$ for each line $l^{\prime}$ parallel to $l$ which is different from $g$. As $|\Pi(g)|=n$, it follows that there is precisely one line $h$ parallel to $g$ which is not in $\Pi(l)$. Let $l^{\prime} \in \Pi(l), l^{\prime} \neq g, l$. Then $h$ meets $l^{\prime}$ and $Q=h \cap l^{\prime}$ is exceptional as $Q$ is on two different parallels to $g$. It follows that $Q$ is the unique exceptional point on $l^{\prime}$. Similarly $h \cap l$ is the unique exceptional point of $l$. This is impossible as it implies that $Q$ is on $n+1$ lines, namely the line $h$ and $l^{\prime}$ and the $n-1$ lines that join $Q$ to the non-exceptional points of $l$. 


\section{Continuing with the general case}

In this final section we complete the proof of Theorem 6 . It follows from Theorem 23 and Propositions 21,22 that the following assumptions can be made: $\delta \geqslant 3, \delta_{l}>0$ for each line $l$ and $\delta_{P}<\delta$ for each point $P$. We start from the following:

Theorem 24. Assume $l$ is a line such that $\delta_{l} \leqslant \delta$ and $\left(\delta^{2}-1\right)(\delta+1)^{2}<27(n+2) / 16$. Then the following hold:

- The lines of $\Pi(l)$ are pairwise parallel. They all have the same deficiency $\epsilon=\delta_{l}$ and $\Pi(l)=\Pi(h)$ for all $h \in \Pi(l)$.

Proof. Observe $|\Pi(l)|=n-(\delta-\epsilon) \leqslant n$. Use induction on $\epsilon=\delta_{l} \geqslant 0$. Case $\epsilon=0$ follows from Proposition 21 and its proof. Assume $\epsilon>0$ in the sequel. Consider the partition $\Pi(l)=\Pi_{1} \cup \Pi_{2}$ where $\Pi_{1}$ consists of $l$ and the lines $h \neq l$ parallel to $l$ which satisfy $\delta_{h}<(n+1+\epsilon) /(2 \epsilon)$. Lemma 18 implies that the lines of $\Pi_{1}$ are pairwise parallel. The induction hypothesis implies $\delta_{h} \geqslant \delta_{l}$ for all $h \in \Pi_{1}$. The next major claim is that $\Pi_{2}$ is empty.

In order to prove this, let $M$ be the set of points covered by lines of $\Pi(l)$ and $N$ the complement of $M$. Then $|N| \geqslant n(\delta-\epsilon)$ and Lemma 11 shows that $N$ consists of exceptional points. Let $D \subset M$ be the set of points which are on more than one line of $\Pi(l)$. Clearly points of $D$ are exceptional. For $X \in D$, let $d_{X} \geqslant 2$ be the number of lines of $\Pi(l)$ on $X$, and $d=\sum_{h \in \Pi_{2}}|h \cap D|$. We have

$$
n|\Pi(l)|-|M|=\sum_{X \in D}\left(d_{X}-1\right) \geqslant \frac{1}{2} \sum_{X \in D} d_{X}=\frac{1}{2} \sum_{h \in \Pi}|h \cap D| \geqslant d / 2
$$

and

$$
\begin{aligned}
\sum_{h \in \Pi_{2}} \sum_{P \in h \backslash D} \delta_{P} & =\sum_{h \in \Pi_{2}}\left(\delta_{h}-\sum_{P \in h \cap D} \delta_{P}\right) \\
& \geqslant \sum_{h \in \Pi_{2}}\left(\delta_{h}-|h \cap D|(\delta-1)\right) \\
& \geqslant\left|\Pi_{2}\right|(n+1+\epsilon) /(2 \epsilon)-d(\delta-1) .
\end{aligned}
$$

We want to lower bound $\sum_{X \in \mathcal{P}} \delta_{X}$. As points of $N$ are exceptional and therefore have deficiency $\geqslant 1$ and because of Equation (1) we have

$$
\sum_{X \in N} \delta_{X} \geqslant n^{2}-|M| \geqslant(n(n-|\Pi|)+d / 2=n(\delta-\epsilon)+d / 2
$$

Together with the points covered by $\Pi_{1}$ this yields the a priori lower bound

$$
\delta n=\sum_{X \in \mathcal{P}} \delta_{X} \geqslant\left|\Pi_{1}\right| \epsilon+n(\delta-\epsilon)+d / 2
$$


which we use to obtain a weak upper bound on $d$ :

$$
d / 2 \leqslant \epsilon\left(n-\left|\Pi_{1}\right|\right)=\epsilon\left(\delta-\epsilon+\left|\Pi_{2}\right|\right)
$$

Collect now the three contributions to $\sum \delta_{X}$ that we have to obtain a lower bound:

$$
\delta n=\sum_{X \in \mathcal{P}} \delta_{X} \geqslant \epsilon\left|\Pi_{1}\right|+\underbrace{(n(\delta-\epsilon)+d / 2)}_{\text {contribution of } N}+\underbrace{\left|\Pi_{2}\right|(n+1+\epsilon) /(2 \epsilon)-d(\delta-1)}_{\text {from (2) }} .
$$

Equivalently

$$
\delta n \geqslant\left|\Pi_{2}\right|((n+1+\epsilon) /(2 \epsilon)-\epsilon)+\epsilon(n-\delta+\epsilon)+n(\delta-\epsilon)-(d / 2)(2 \delta-3) .
$$

Substitute inequality (3) for $d / 2$. After simplification this yields

$$
\delta n \geqslant\left|\Pi_{2}\right|((n+1+\epsilon) /(2 \epsilon)-2 \epsilon(\delta-1))+\delta n-2 \epsilon(\delta-1)(\delta-\epsilon) .
$$

Because of the hypothesis in Theorem 24 the coefficient of $\left|\Pi_{2}\right|$ is positive. Assume $\left|\Pi_{2}\right| \geqslant 1$. Then

$$
n+1+\epsilon \leqslant 4(\delta-1) \epsilon^{2}(\delta-\epsilon+1) .
$$

The left is $\geqslant n+2$, the right side is maximized by $\epsilon=2(\delta+1) / 3$ as a function of $\epsilon$. This yields $n+2 \leqslant(16 / 27)\left(\delta^{2}-1\right)(\delta+1)^{2}$ contradicting the hypothesis.

Now $\Pi=\Pi_{1}$ consists of $n-\delta+\epsilon$ parallel lines, each of deficiency $\geqslant \epsilon$.

Finally we prove that $\Pi(l)=\Pi(h)$ for all $h \in \Pi(l)$, equivalently: each line in $\Pi(l)$ has deficiency $\epsilon$. The situation is: $\Pi(l)$ has $n-\delta+\epsilon$ lines. They are pairwise parallel, have deficiency $\geqslant \epsilon$. Since the $n(\delta-\epsilon)$ points not covered by the lines of $\Pi(l)$ have positive deficiency, then global counting shows

$$
\delta n=\sum_{P \in \mathcal{P}} \delta_{P} \geqslant \epsilon(n-\delta+\epsilon)+n(\delta-\epsilon)
$$

which shows that at most $z=\epsilon(\delta-\epsilon) \leqslant \delta^{2} / 4$ lines of $\Pi(l)$ have deficiency $>\epsilon$. Clearly $\Pi(l)=\Pi(h)$ for all $h \in \Pi(l)$ of deficiency $\epsilon$.

Assume there is some $g \in \Pi(l)$ of deficiency $>\epsilon$. Then $g$ is parallel to some line $h$ which is not in $\Pi(l)$. Let $l=l_{1}, \ldots, l_{c}$ be the lines of $\Pi(l)$ of deficiency $\epsilon$. We saw above that

$$
c \geqslant|\Pi|-z=n-(\delta-\epsilon)(\epsilon+1) \geqslant n-(\delta+1)^{2} / 4 .
$$

Then $h$ meets each of the $l_{j}$.

Each point of $h$ is exceptional. Indeed, if point $X$ of $h$ lies on no line of $\Pi(l)$ it is exceptional for this reason, and if $X$ is on a line of $\Pi(l)$, then it lies on two parallels to $g$ and is therefore exceptional.

It follows in particular $\delta_{h} \geqslant n$. Line $h$ is parallel to $n-1-\delta+\delta_{h} \geqslant 2 n-1-\delta$ other lines. At most $z$ of those are in $\Pi(l)$. Let $s=2 n-1-\delta-z \geqslant 2 n-(\delta+2)^{2} / 4$ and $h_{1}, \ldots, h_{s}$ lines parallel to $h$ which are not in $\Pi(l)$. Count the ordered pairs of different 
$h_{i}$. There are $s(s-1)$ such ordered pairs. Consider the lines $l_{j} \in \Pi(l), j=1, \ldots c$. Each $l_{j}$ contains $m_{j} \leqslant \epsilon-1 \leqslant \delta-1$ exceptional points not on $h$ and $n-1-m_{j}$ non-exceptional points. The non-exceptional points of $l_{j}$ lie on at most one line parallel to $h$ and thus on at most one line $h_{i}$; thus at least $s-\left(n-1-m_{j}\right)$ lines $h_{i}$ must meet $l_{j}$ in one of the $m_{j}$ exceptional points not on $h$. The Cauchy-Schwartz inequality shows that the number of pairs of different $h_{i}$ meeting in a point of $l_{j}$ is at least

$$
m_{j} \frac{s-n+1+m_{j}}{m_{j}}\left(\frac{s-n+1+m_{j}}{m_{j}}-1\right) \geqslant \frac{(s-n+1)^{2}}{\delta-1} .
$$

We obtain the inequality

$$
c(s-n+1)^{2} \leqslant(\delta-1) s(s-1) .
$$

Let us create a factor of $s$ on the left side: as $s \leqslant 2(n-1)$ we obtain $(n-1)^{2} \geqslant(n-1) s / 2$ and $(s-(n-1))^{2}=s^{2}-2(n-1) s+(n-1)^{2} \geqslant s(s-2(n-1)+(n-1) / 2)$. The major inequality simplifies after factoring $s$ :

$$
c(s-3(n-1) / 2) \leqslant(\delta-1)(s-1)<(\delta-1) 2 n .
$$

Using the lower bounds:

$$
(\delta-1) 2 n>\left(n-(\delta+1)^{2} / 4\right)\left((n+3) / 2-(\delta+2)^{2} / 4\right),
$$

after multiplying out on the right, forgetting the terms not dependent on $n$ and canceling the factor $n: 2(\delta+2)^{2}+(\delta+1)^{2}+16(\delta-1)>4 n$ which clearly contradicts the hypothesis.

Theorem 25. Let $\left(\delta^{2}-1\right)(\delta+1)^{2}<27(n+2) / 16$. Then every line has deficiency $\leqslant \delta$.

Proof. Pick a non-exceptional point $P_{0}$ and its bundle of $n+1$ lines $l_{1}, \ldots, l_{n+1}$. Let $\delta_{i}=\delta_{l_{i}}$ and $\epsilon_{i}=\delta-\delta_{i}$. We want to show that $\epsilon_{i} \geqslant 0$ for all $i$. As every line is parallel to one of the lines $l_{i}$, the preceding theorem then implies the statement.

Assume therefore that some of the $\epsilon_{i}$ are negative. As $\sum \epsilon_{i}=\delta$ and at least one negative $\epsilon_{i}$ is present, we can find some positive $\epsilon_{i}$ that sum to at least $\delta+1$. As $\epsilon_{i} \leqslant \delta$ for all $i$ we can choose them such that the sum is at most $2 \delta$. Choose therefore the lines $l_{1}, \ldots, l_{s}$ such that $\epsilon_{i} \geqslant 0$ for $i \leqslant s$ and $z=\sum_{i=1}^{s} \epsilon_{i}$ satisfies $\delta+1 \leqslant z \leqslant 2 \delta$. Use the result of the preceding theorem: let $\Pi_{i}=\Pi\left(l_{i}\right)$ for $i \leqslant s$ and recall that $\Pi_{i}$ consists of $n-\epsilon_{i}$ parallel lines each of which has deficiency $\delta_{i}$. Let $M_{i}$ be the set of points covered by the lines of $\Pi_{i}$ and $N_{i}$ its complement. We have $\left|M_{i}\right|=n\left(n-\epsilon_{i}\right)$ and $\left|N_{i}\right|=n \epsilon_{i}$. Also, let $N=\cup_{i=1}^{s} N_{i}$. Observe that $N$ consists of exceptional points. Recall also from Theorem 24 that lines $l \in \Pi_{i}, g \in \Pi_{j}$ for $i<j \leqslant s$ must meet. This has the following evident consequences: if $l \in \Pi_{i}$ and $i \neq j$, then $\left|l \cap M_{j}\right|=n-\epsilon_{j}$ and $\left|l \cap N_{j}\right|=\epsilon_{j}$. It follows $\left|N_{i} \cap N_{j}\right|=\epsilon_{i} \epsilon_{j}$.

Here is a first lower bound on $|N|$ : observe at first that $|N| \geqslant \sum_{i=1}^{s}\left|N_{i}\right|-\sum_{i<j \leqslant s} \mid N_{i} \cap$ $N_{j} \mid$. In fact, the right side does count elements of $N$. If $P \in N$ occurs in precisely $t \geqslant 1$ of 
the $N_{i}$, then the contribution of $P$ to the right side is $t-\left(\begin{array}{l}t \\ 2\end{array}\right) \leqslant 1$. The inequality follows. Continuing on the right side we obtain

$$
|N| \geqslant \sum_{i=1}^{s} \epsilon_{i} n-\frac{1}{2} \sum_{i, j=1}^{s} \epsilon_{i} \epsilon_{j}=z n-(1 / 2) z^{2}
$$

Consider again a point $X \in N$. It is exceptional and therefore on at most $n$ lines. Let $X$ be in $N_{i}$ for $i \leqslant t$ and in $M_{i}$ for $t<i \leqslant s$. The number of points of $N$ which are collinear with $X$ is bounded by

$$
\sum_{i=1}^{t} n\left(\epsilon_{i}-1\right)+\sum_{i=t+1}^{s} n \epsilon_{i}=n z-n t .
$$

Together with Equation (4) this shows that the number of points of $N$ which are not collinear with $X$ is $\geqslant|N|-1-n z+n t \geqslant n t-1-z^{2} / 2$. As $z \leqslant 2 \delta$ it follows that the number of points in $N$ not collinear with $X$ is $\geqslant t n-1-2 \delta^{2}$ and therefore $>(t-1) n$. We conclude $\delta_{X} \geqslant t$. Finally $\sum_{X \in N} \delta_{X}$ is lower bounded by the number of pairs $(i, X)$ where $i \leqslant s$ and $X \in N_{i}$. This number is $\sum_{i=1}^{s}\left|N_{i}\right|=\sum \epsilon_{i} n>\delta n$. As $\sum_{X \in N} \delta_{X} \leqslant \delta n$ by Lemma 12 , this is a contradiction.

We are ready for the final step.

Theorem 26. Each $P(n, \delta)$ is embeddable provided $\left(\delta^{2}-1\right)(\delta+1)^{2}<27(n+2) / 16$.

Proof. Assume $\left(\delta^{2}-1\right)(\delta+1)^{2}<27(n+2) / 16$. We need to show that $P(n, \delta)$ can be embedded in a $P(n, \delta-1)$. As before start from a non-exceptional point $P$ and its bundle of lines. As $\sum \delta_{i}=\delta n$ it follows that there is some line $l_{1}$ on $P$ whose deficiency is $d<\delta$. Let $\Pi=\Pi\left(l_{1}\right)$, a parallel class of $n-\delta+d$ lines, each of deficiency $d$. Let $M$ be the set of points covered by $\Pi$, and $N$ the complement of $M$. Assume all $X \in N$ satisfy $\delta_{X} \geqslant 2$. An obvious count yields the contradiction $\delta n=\sum_{Q} \delta_{Q} \geqslant d(n-\delta+d)+2 n(\delta-d)$, equivalently $(n-d)(\delta-d) \leqslant 0$. It follows that there is some $X \in N$ such that $\delta_{X}=1$. Let $U$ be the union of $X$ and the $n-1$ points not collinear with $X$. Assume some line $l$ contains at least two points of $U$. Let $t=|l \cap U| \geqslant 2$. Then there are some two lines on $X$ which are both parallel to $l$. We are in the situation of Lemma 18 which, in conjunction with Theorem 25, implies $n+1 \leqslant \delta(2 \delta-1)$. This contradiction shows that $U$ can be used as a new line which together with the lines of the $P(n, \delta)$ forms a $P(n, \delta-1)$. This completes the proof.

\section{References}

[1] J. Bierbrauer, S. Black and Y. Edel: Some t-homogeneous sets of permutations, Designs, Codes and Cryptography 9 (1996), 29-38.

[2] J. Bierbrauer and Y. Edel: Theory of perpendicular arrays, Journal of Combinatorial Designs 6 (1994), 375-406. 
[3] M. Bogaerts: Isometries and construction of permutation arrays, IEEE IT Transactions 56 (2010), 3177-3179.

[4] R. H. Bruck: Finite nets II. Uniqueness and embedding, Pacific J. Math. 13 (1963), 421-457.

[5] W. Chu, C. Colbourn, P. Dukes: Constructions for permutation codes in powerline communications, Designs, Codes and Cryptography 32 (2004), 51-64.

[6] H.C. Ferreira, A.J.H. Vinck: Inference cancellation with permutation trellis arrays, Proc. IEEE Vehtcular Technology Conf. 2000, 2401-2407.

[7] S. Huczynska: Powerline communication and the 36 officers problem, Phil. Trans.R. Soc.A 364 (2006), 3199-3214.

[8] I. Janiszczak, R. Staszewski: An improved bound for permutation arrays of length 10, manuscript.

[9] T. Kløve, Classification of permutation codes of length 6 and minimum distance 5, Internat. Symposium on Info. Theory and its Applications, Honolulu 2000, 465-468.

[10] T. Kløve: A combinatorial problem motivated by a data transmission application, Chapman and Hall/ CRC Press 2004.

[11] Bill Martin and B.E. Sagan: A new notion of transitivity for groups and sets of permutations, Journal of the London Mathematical Society 73 (2006), 1-13.

[12] K. Metsch: Improvement of Bruck's completion theorem, Designs, Codes and Cryptography 1 (1991), 99-116.

[13] J. Quistorff: A survey on packing and covering problems in the Hamming permutation space, Electron. J. Comb. (2006), A1.

[14] D.R. de la Torre, C.J. Colbourn, A.C.H. Ling: An application of permutation arrays to block ciphers, Congr. Numer. 145 (2000), 5-7.

[15] A.J. Han Vinck: Codes modulation for powerline communications, AE Internat. Journal of Electronics and Commun. 54 (2000), 45-49. 